\title{
Study on the Evaluation System of Slope Stability Analysis Based on GIS and Its Application
}

\author{
Junlan Zhao ${ }^{*}$ \\ College of Civil Engineering, North China University of Technology, Beijing, 100144, China
}

\begin{abstract}
The application of GIS to the forecast and evaluation in the regional landslide can provide scientific basis for the regional geological environment management and the geological disaster assessment, can integrate the scattered data to share each other, and can improve data value. Its powerful interface function library provides a good tool for the professional GIS design and development. This study carried out rapid modeling, efficient calculation and strong visibility in the slope evaluation by highly fusing Geographical Information Systems (GIS) and slope engineering together and combining Supermap GIS with ANSYS, which are representative software in their fields. Furthermore, the system of slope stability analysis and evaluation was established by quadric developing, which made a bridge between the fields of GIS and slope stability assessment.
\end{abstract}

Keywords: GIS, fuzzy comprehensive evaluation, finite element, ANSYS.

\section{INTRODUCTION}

In recent years, the development of GIS technology has injected a new vitality for slope stability analysis and made the latter showing a new trend [1]. The GIS powerful capabilities for database management and spatial analysis can greatly improve disaster prediction accuracy and shorten evaluation cycles, so that can provide timely decision support for the slope engineering problems encountered in the regional economic construction. Comprehensively reviewing the previous researches, most studies analyzed qualitatively the relationship between slope failure and various influencing factors only using statistical method, and made the slope hazard maps based on the spatial analysis function of GIS. There are a few researchers who used quantitative mechanical model, but also limited the use of infinite slope model. Xie Mo-wen, etc. studied the three-dimensional slope and its risk analysis. In this paper, based on previous research progress, the slope stability was assessed by using comprehensively qualitative evaluation and numerical analysis methods and made some practical results.

\section{QUALITATIVE EVALUATION BASED ON FUZZY COMPREHENSIVE EVALUATION}

For evaluating something, we often involve in multiple factors or multiple indicators. To quantitate indicator information suing the fuzzy approach, then to evaluate qualitatively and make decision for multivariate, this is the fuzzy comprehensive evaluation. Because the slope stability was affected by a lot of factors and the traditional quantitative assessment methods cannot fully consider the uncertainty actually existed, the given safety factor cannot fully reflect the true safety and reliability [2]. In order to reflect the true uncertainty of the slope and get the slope stability evaluation closer to the true situations, we made the comprehensive evaluation model using fuzzy method.

\subsection{Algorithm Principle}

The mathematical models of Fuzzy comprehensive evaluation are divided into single-stage and multi -stage model. We can use single -level model when there are only a few evaluated factors. When there are too many factors to be evaluated, too much information is often filtered in the process of "taking small" or "taking big", so that the result is completely useless in single-factor judge. Because there are many factors affected slope stability, a reasonable allocation of right number is difficult, namely the truly effect of each factor in the whole cannot be reflected. In this time, we need to adopt a multi -level evaluation [3].

Principle is as follows:

First, determine the rating scale vector $V=\left\{v_{1}, v_{2}, \cdots, v_{n}\right\}$ ( $n$ is the number of rating scale) and evaluation factor vector $U=\left\{u_{1}, u_{2}, \cdots, u_{m}\right\}$ ( $m$ is the number of factors affecting).

The univariate evaluation subset of $u_{i}$ is $R_{i}=\left\{r_{i 1}, r_{i 2}, \cdots, r_{i n}\right\}$, make $m$ - related factors to constitute a total evaluation matrix $R$ :

$$
\mathrm{R}=\left[\begin{array}{ccc}
\mathrm{r}_{11} & \cdots & \mathrm{r}_{1 \mathrm{n}} \\
\vdots & \ddots & \vdots \\
\mathrm{r}_{\mathrm{m} 1} & \cdots & \mathrm{r}_{\mathrm{mn}}
\end{array}\right]
$$

In the formula: $r_{i j}=\mu\left(u_{i}, v_{j}\right),\left(0 \leq r_{i j} \leq 1\right)$ show the membership of evaluation factor $u_{i}$ belong to the evaluation 
level $v_{j}$. Given the weight vector $A$ (different importance of fuzzy subsets of characterization of the various factors associated with the evaluation of things or property):

$$
A=\left(a_{1}, a_{2}, \ldots, a_{m}\right) 0 \leq a_{i} \leq 1, i=1,2, \ldots, m
$$

In the formula: $a_{i}$ is the membership of $u_{i}$ to $A$, is a measure of the degree of influence on the overall evaluation of single factor of $u_{i}, \sum_{i=1}^{m} a_{i}=1$

The evaluation set

$$
B=\left(b_{1}, b_{2}, \ldots, b_{n}\right) \quad\left(0 \leq b_{j} \leq 1\right) \text {, the fuzzy evaluation }
$$
expression is:

$$
B=A \times R \text { or } b_{j}=\sum_{i=1}^{m} a_{i} r_{i j}
$$

Finally, according to the principle of maximum membership degree $\operatorname{Max}\left(b_{j}\right)$ correspond level $v_{j}$ to determine the results of evaluation unit.

In this evaluation of slope stability, evaluation level vector represents the degree of slope stability classification:

$V=($ Stable, Relatively stable, Less stable, Unstable $)$

Evaluation factors vector represents the slope stability evaluation:

$$
U=\left(\begin{array}{l}
\text { Cohesion , Internal friction angle , Slope angle , slope height , } \\
\text { The maximum seismic intensity }
\end{array}\right.
$$

Vector A is weight vector of slope stability calculated by excessive weighting method. Determination of fuzzy relation matrix $\mathrm{R}$ and the weight vector $\mathrm{A}$ is an important part of the fuzzy evaluation. Among them, determine the elements $r_{i j}$ in the relationship matrix $R$ can use information distribution method [4] or constructed membership function method [5].

\subsection{Fuzzy Evaluation Method Based on GIS}

The fuzzy evaluation method based on GIS is to effectively combine the digital maps of study area with the judgment mathematical model, to complete the security classification of study area by operating graphics. This paper selected slope of Sichuan Saide as an example, the specific process is as follows:

1) According to the specific circumstances of the judge region, the main evaluation index system is selected as shown in Fig. (1).

2) Map digitization. Put contour map and drill point layout of the area into the GIS system, then convert them into contour layers and drill point layer. These layers include the underlying property and elevation fields corresponding to the drilling points.

3) By clicking geometric objects on the layer, the measured value of evaluation index can be extracted and calculated in the mathematical model. The calculation results as attribute field "JudgeResult" save in the attribute table.
4) Interpolation calculation is done for drilling points which are discrete and uneven distribution. The conversion process of "from the point to the surface" is carried out by taking "JudgeResult" as the difference field. The rating evaluation results of regional stability are obtained finally.

\section{TWO AND THREE-DIMENSIONAL MODELING IN GIS}

The digital elevation model (DEM) in GIS is commonly used to express the undulating status of terrain surface.

Digital elevation model achieve the simulation of surface topography by using the limited digital terrain elevation data, in other words, it is the digital expression of the terrain surface morphology, abbreviated to DEM (Digital Elevation Model)0. Application of DEM in terrain analysis is focused on 3D drawing, ground dizziness drawing, vision analysis, and terrain analysis and so on [6].

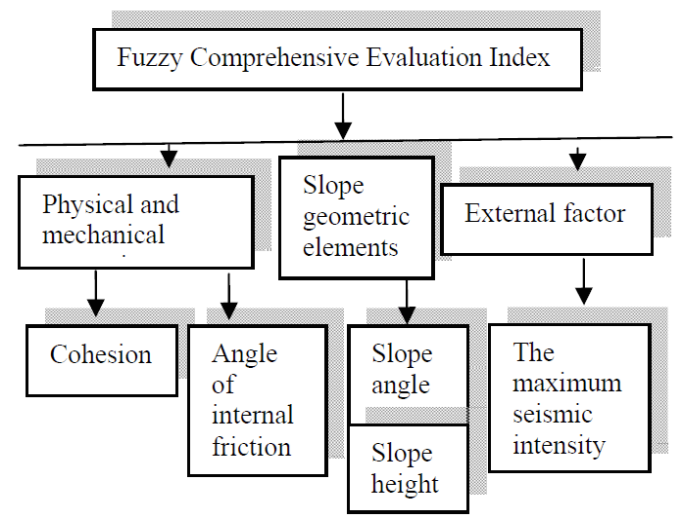

Fig. (1). Evaluation index system.

\subsection{Tin Model and Grid Model}

DEM model based on the Triangulated irregular network (TIN) represents the terrain surface by using a series of triangles which are non cross, no overlapping and connected together.

Raster digital elevation (Grid DEM) model, corresponding to GIS raster data structure, divide the study area into limited small squares finite element (equal to the numbers of ranks and files), in which plane coordinate of grid intersection are implied in rank and file numbers of the grid. The grid points can be seen as a matrix or array in Mathematics. Each grid unit has a value (typically elevation, may be other attribute values), which corresponds to each element of the matrix. Raster data structure can be processed and applied easily by computer [7].

The GIS models involved in this paper usually are raster data models, which have advantages of high accuracy, good visual effects, simple algorithm, and easy conversion and so on.

\subsection{Conversion from the Tin to the Grid}

Conversion to the grid model from the TIN is actually based on the interpolation calculation of the TIN. This study carried out interpolation calculations for discrete point data by using Kriging interpolation method. 
The basic principle of Kriging interpolation method is similar to the least squares method. Its essence is to consider the general variation trend of the sampling surface, (statistical characteristics of the surface), also consider relevant and random changes in sampling surface topography.

The mathematical formula is 0 :

$f(x, y)=f_{1}(x, y)+f_{2}(x, y)+C$

Of which: $f_{1}(x, y)$ is the structural item, used to express the overall trend of terrain surface; $f_{2}(x, y)$ is the relevant item, used to express the relevant changes of terrain surface; $C$ is random noise, used to express the random variation of the terrain.

\subsection{Generation of 3D Perspective View}

Three-dimensional topographic map can effectively reflect the three-dimensional view of the terrain. DEM model is the basis of a three-dimensional topographic map. The three-dimensional topographic map can be generated in GIS according to the discrete vector points. The generation process is shown in Fig. (2), the result shown in Fig. (3).

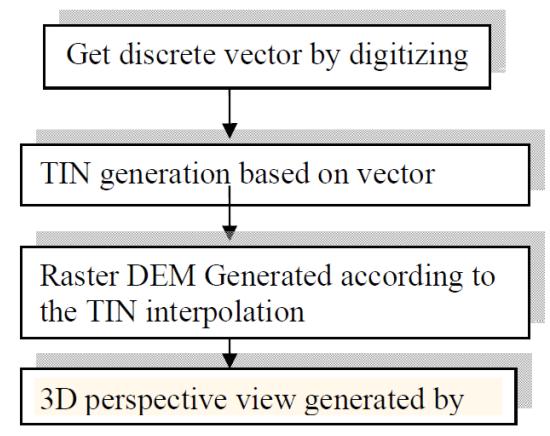

Fig. (2). Process of generating 3D topographic map.

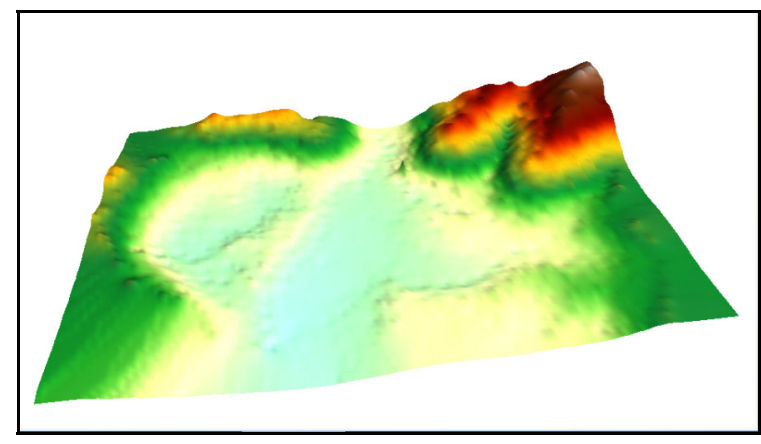

Fig. (3). Generated 3D topographic map.

\subsection{Extraction of GIS Stratigraphic Section}

For digitized contour lines and drilling point data, how to quickly generate slope cross-section became the priority problem of slope stability calculation. In this study, using the characteristics of point, line, surface data structures in GIS, an algorithm to automatically generate cross-sectional view according to the drilling point data is designed. Slope sections are created successively in order of cross-sectional point, line and surface.

\section{DEVELOPMENT AND INTEGRATION OF THE SYSTEM}

\subsection{System Design}

Software system in this paper include: GIS basic functional module, management module of data query, qualitative analysis module, 2D, 3D modeling of slope and visualization ANSYS interface module and display module, as shown in Fig. (4). Main interface is shown in Fig. (5).

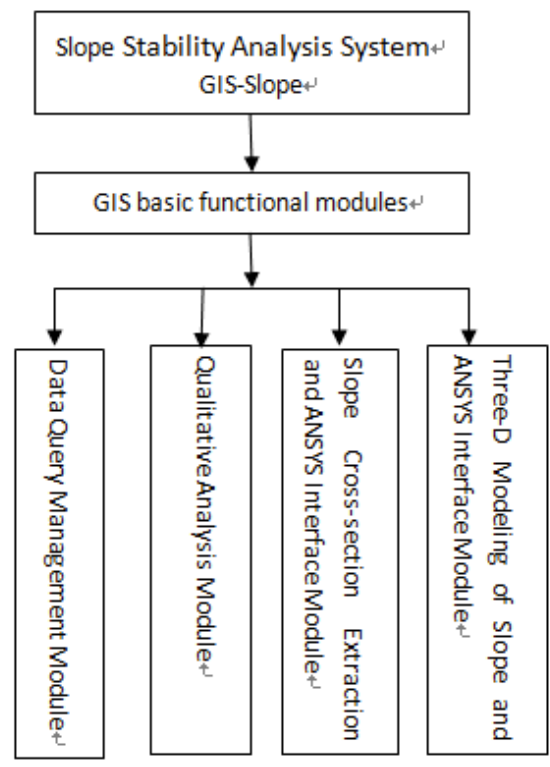

Fig. (4). Diagram of system design.

\subsection{GIS Basic Functional Modules}

In order to achieve the advanced features of subsequent computational analysis, we need to design and implement some basic functions of GIS, such as the map to zoom, pan, and rotate and so on; bidirectional query of spatial data and attribute data; addition, deletion, CAD drawings, layout and printing of maps on datasets.

\subsection{Management Module of Data Query}

This module is responsible for displaying map data, monitoring data, and interactive query spatial / attributes data and other functions. In the query interface, a gray prediction function is also provided for deducting displacement data of monitoring points.

\subsection{Fuzzy Comprehensive Evaluation Module}

This module can select the evaluation factor values according to the recorded geological data based on the extracting slope section or three-dimensional model, so that we can achieve fast stability fuzzy evaluation.

Object of the fuzzy comprehensive evaluation method is a region rather than a specific point or section, therefore, the ultimate goal of this program is to get the judged results of the entire study area. Based on the idea of discrete data to be rasterized, taking the evaluation value of a single point as the interpolation field (equivalent to the elevation field in DEM), the grid map of fuzzy comprehensive evaluation in the re- 


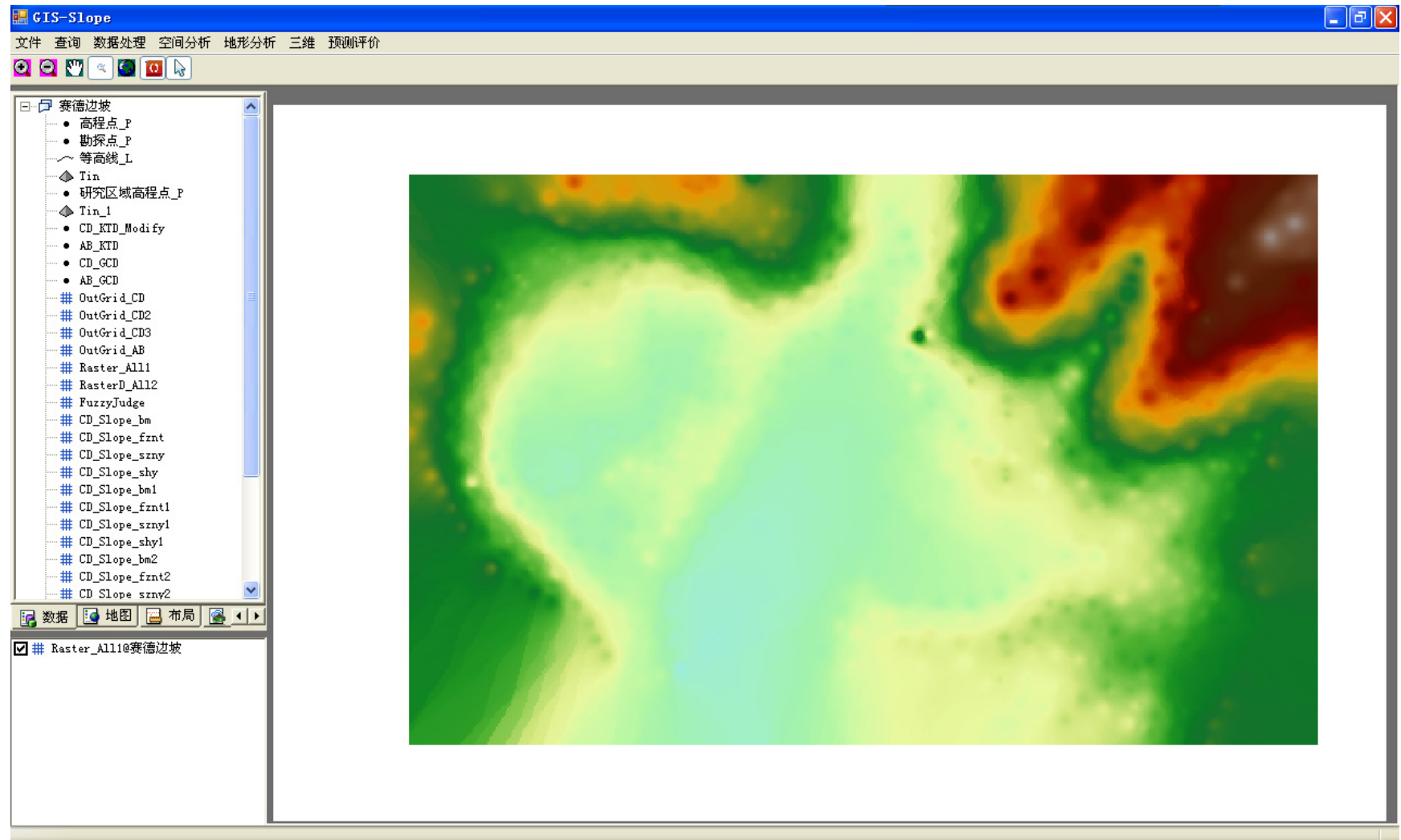

Fig. (5). Main interface of system.

gional slope stability can be generated by using Kriging algorithm.

\subsection{Two-Dimensional Analysis Modules}

In the process of preparing the FEM interface, the corresponding algorithms is designed to improve the intelligence process, including GIS module passed formation parameters to the finite element module; the profile model in GIS ( point, line, polygon data set ) converted to a geometric model can be called by ANSYS; automatic search model border.

\subsubsection{Geometric Model Conversion}

The spatial data in GIS is saved in the form of point, line and polygon data set. In order to convert geometric data from GIS to ANSYS, we need to extract and convert respectively for different data sets.

\subsubsection{Extract Boundary and Define the Constraints}

In pre-treatment process of the finite element, we need to restrict the freedom of the slope boundary, namely to set as 0 for each node in the $\mathrm{x}$ direction displacement of slope hind boundary and $\mathrm{x}, \mathrm{y}$-direction displacement of the bottom boundary. This requires that the program be able to automatically search and extract the slope boundary lines.

The above operation is actually the process to write the code in the ANSYS command stream file. After above operation, an ANSYS macro file (*. Mac file) can be generated. When entering the ANSYS operating environment, you can use "/ INPUT" command to directly carry out calculation.

\subsection{Three-Dimensional Analysis Modules}

The center of each grid in the generated raster graphics is extracted as the key (KeyPoints) of ANSYS model. It should be noted that the process of extracting the center of the grid must be ordered, that is according to the first row or the first column.

\section{APPLICATION TO ENGINEERING}

\subsection{Overview of Project Site}

The site is located in the low mountains of southern $\mathrm{Si}$ chuan, where a cement clinker production line $(4600 \mathrm{t} / \mathrm{d})$ with energy-saving and environmental protection will be built. The north, east and south of proposed site are surrounded by mountains, only south-west side is the concave as exit. Proposed site is located in mountain slopes and valley with height above sea level $659 \mathrm{~m}-805 \mathrm{~m}$, relative height distance is $146 \mathrm{~m}$. Rock occurrence main towards to east-west, outcrops are mainly limestone, sandy mudstone and muddy sandstone and swelling clay. The slope stability will be affected greatly by the construction in the site. These slopes had adverse combination of structural surface will produce geological disasters such as landslides, avalanches and so on under imbalance of stress conditions.

\subsection{Regional Stability Evaluation}

The fuzzy comprehensive evaluation of explored points in the region was carried out point by point. The results were added to the attribute table. The results were rasterized and generated regional stability classification diagram. Color 


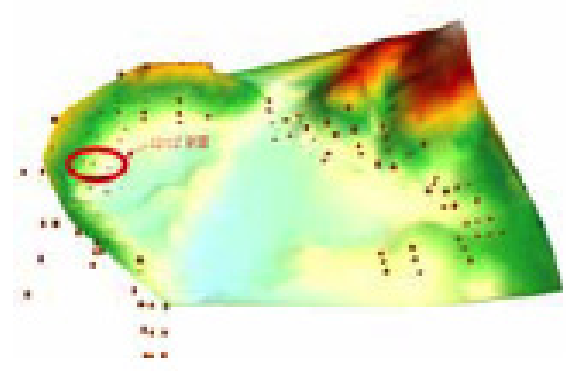

a

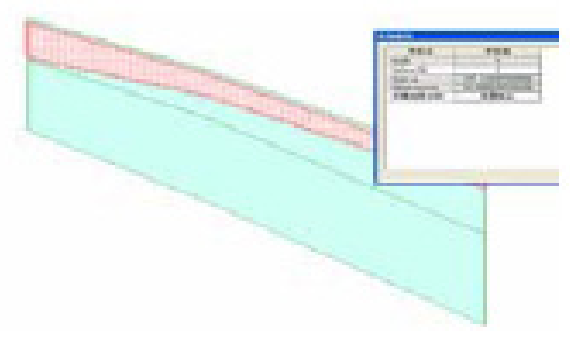

C

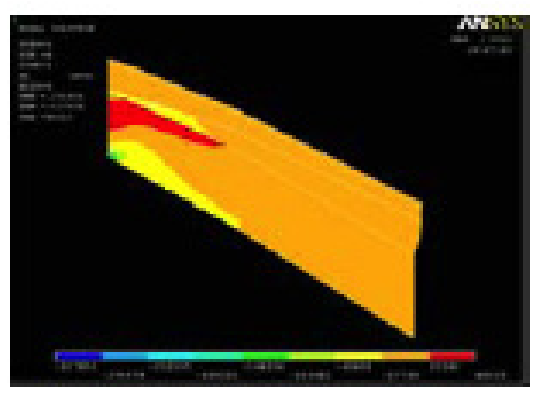

b

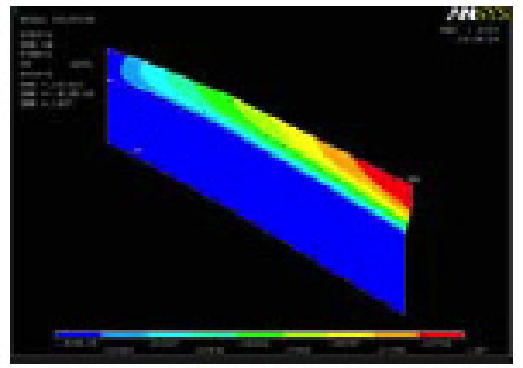

d

Fig. (6). 2D section of slope analysis. (a) Extraction of Engineering Slope sectional analysis; (b) Sectional program interface; (c) Total strain diagram of Cross-section; (d) Total cross-sectional diagram of stress.

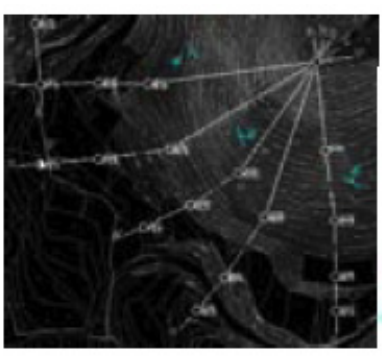

a

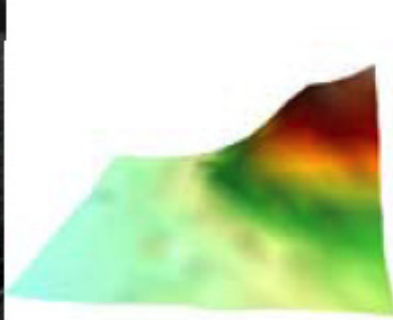

$\mathrm{b}$

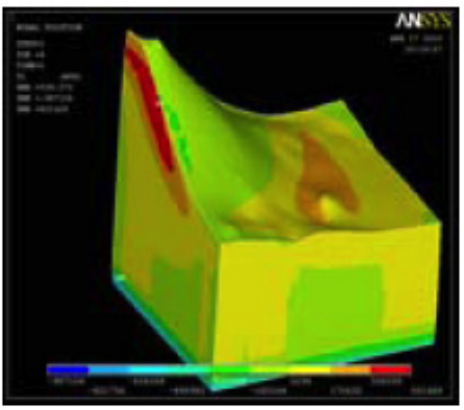

$\mathrm{d}$

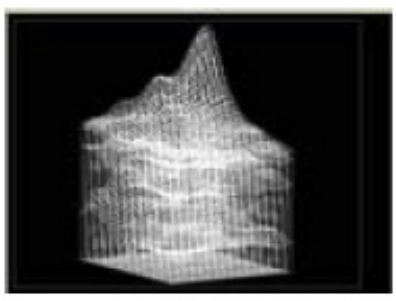

$\mathrm{c}$

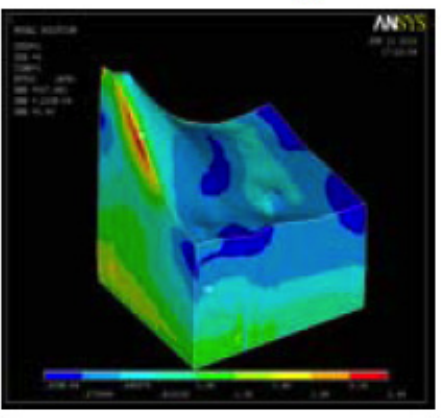

e

Fig. (7). 3D analysis of slope. (a) Geological map of slope engineering; (b) 3D map of Engineering Slope ; (c) Raster of the slope plane ; (d) The results of 3D slope stability analysis; (e) The results 3D slope stability analysis.

from green to red represent stable, more stable, more volatile and unstable four levels. In order to present grading results more continuously, we can appropriately increase the number of classification and raster precision. In addition, the system can also superimpose raster datasets over 3D graphics to maximize visualization capabilities of GIS. The evaluation results shown, the red zone occurred at west, northeast and east in the project site, where the slopes were more dangerous and should be managed. To get accurate results, we need to add qualitative calculation and analysis of the monomer slope. 


\subsection{D Cross-Sectional Analysis of Slope}

The profile section of 12-12 Slope geological map of the site is extracted by using stratigraphic section extraction module. Fig. (6a) shows the location of 12-12 ' section in the site map (shown in red arrows). We selected the three drill point referred by the red arrow (ZK11, ZK326 and ZK325) to extract. The profile section extracted by procedure is shown in Fig. (6b). To click the below can display its attribute data. The total cross section and the total stress strain diagrams are shown in Figure $(\mathbf{6 c}) \&(\mathbf{6 d})$.

The practice proved that the method extracted profile sections by the GIS and endowed with the attribute, and then calculated by ANSYS can maximally reduce the modeling time in finite element analysis. The advantages of this method will be more obvious for the object need to be repeated study, such as calculation of slope safety factor by using strength reduction.

\subsection{D Slope Stability Analyses}

CD Slope of project site was selected for 3D analysis, which is located in the northeast corner of the site. The slope length is $256 \mathrm{~m}$, slope height $78 \sim 80 \mathrm{~m}$, the slope digress 35 $\sim 50^{\circ}$, slope aspect $261^{\circ} \sim 326^{\circ}$. The ground filled with soil tillage, clay, gravel, clay and shale sandstone. The average production -like is $100^{\circ} 45^{\circ}$, belonging to rock slope. The exploration point arrangement of the slope was shown in Fig. (7).

Elevation point data was extracted from the overall plan of the region, and flat raster and 3D perspective view were generated in turn, as shown in Fig. (7a), (7b), (7c). Calculation results are shown in Fig. (7d), (7e).

Fig. (7d) shown that the slope area maximum of the first principal stress occurred in the red zone at the waist; the main stress of orange area at the foot was also larger. Since the analysis was carried out in the gravity field, the result of analysis was more identical with the actual situation. Fig. (7e) shown the strain field, and its distribution broadly consistent with Fig. (7d), but the plastic strain is small, which indicated that the segment slope should be stable on the whole. The result was proven to be identical with that of the stereographic projection calculations.

\section{CONCLUSION}

1) The fuzzy comprehensive slope stability evaluation system was established by using raster data modeling based on combining the fuzzy comprehensive evaluation method with GIS.
2) Through the study of GIS vector, raster data models and data storage format of ANSYS software, we designed interface program, established a method of ANSYS slope simulation model, and completed the data conversion between GIS and ANSYS.

3) The slope analysis and evaluation system was designed by integrated application of database technology and GIS component technology, which can achieve the functions of the slope modeling, analysis, calculation and evaluation.

GIS slope research is a complex project, which is involved in not only the stability analysis mathematics and mechanics of slope engineering, but also knowledge of GIS and computer-related disciplines. This paper implements the full integration of GIS and the finite element calculation. The evaluation system need to be further improved for the deformation instability prediction and warning of complex slope engineering, especially for the precise threedimensional terrain modeling of the complex formation and fusion with classical mechanics models.

\section{CONFLICT OF INTEREST}

The author confirms that this article content has no conflict of interest.

\section{ACKNOWLEDGEMENTS}

The paper is funded by the special scientific research and reform project of promoting talent training: Study on soilcompacting effects induced by earth pressure balance shield machine cutter head and their simulation (14085-11).

\section{REFERENCES}

[1] Z. Q. Cui, and N. Li, "Slope engineering", Water Power Press, pp. 32-33, Beijing, 1999

[2] C.Y. Zhang, Y. X. Gao, and M. F. Jiang, "Two fuzzy comprehensive evaluation in highway slope stability analysis applications," Highway Engineering, vol. 33, no. 5, pp. 109-113,2008.

[3] J. D. Wang, X. C. Feng, and M. X. Min, "Fuzzy Information Analysis of loess slope stability”, Mountain Research, vol. 9, no. 1, pp. 33-40, 1991.

[4] Z. R. Liu, "The Analysis of Fuzzy Mathematical Method, Fuzzy Math in Earthquake Research", Seismological Press, pp. 95-106, Beijing 1985.

[5] J. Zhang, N. W. Kui, and X. J. Feng, "Two fuzzy comprehensive evaluation of Yan'an City slope stability," Journal of Xi'an Institute of Geology, vol. 12, no. 1, pp. 82-91, 1990.

[6] P. G. Du, "Mechanical design and manufacturing", Mechanical Industry Press, pp. 126-135, Beijing, 2005.

[7] X. Li, and K. Liu, "GIS and spatial analysis principles and methods”, Science Press, pp. 73-75, 2006. 